\title{
Diacronie
}

Studi di Storia Contemporanea

$\mathrm{N}^{\circ} 32,4 \mid 2017$

Proiezioni individuali e agire collettivo nella storia

\section{Introduzione. Laboratorio n. 32 - dicembre 2017}

\section{Eloisa Betti e Maria Pia Casalena}

\section{(2) OpenEdition}

\section{Journals}

\section{Edizione digitale}

URL: http://journals.openedition.org/diacronie/6435

DOI: $10.4000 /$ diacronie. 6435

ISSN: 2038-0925

\section{Editore}

Association culturelle Diacronie

Notizia bibliografica digitale

Eloisa Betti e Maria Pia Casalena, « Introduzione. Laboratorio n. 32 - dicembre 2017 », Diacronie [Online], N 32, 4 | 2017, Messo online il 29 décembre 2017, consultato il 24 septembre 2020. URL : http://journals.openedition.org/diacronie/6435; DOI : https://doi.org/10.4000/diacronie.6435

Questo documento è stato generato automaticamente il 24 settembre 2020.

Creative Commons License 


\section{Introduzione. Laboratorio n. 32 - dicembre 2017}

Eloisa Betti e Maria Pia Casalena

Il lavoro delle donne: temi, dibattiti, culture 
1 Il filo rosso che lega i saggi contenuti in questo dossier è il lavoro delle donne. Gli elaborati sono stati realizzati da un gruppo di studenti della laurea magistrale in Scienze Storiche e Orientalistiche dell'Università di Bologna, nell'ambito del corso di Storia sociale e di genere tenuto da Maria Pia Casalena (a.a. 2016-17) e, in

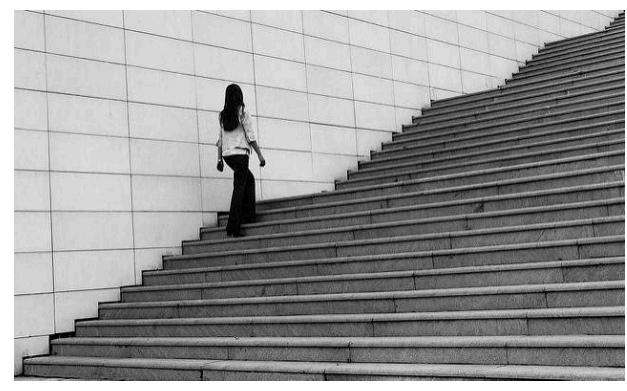
particolare, del seminario "Ripensare la questione sociale: un approccio di genere" curato da Eloisa Betti.

2 Un secolo di lavoro femminile, dagli anni Dieci del Novecento a oggi, è stato analizzato mettendo a fuoco diversi temi, dibattiti e culture. I contributi prendono in esame fenomeni di portata globale, come la precarietà del lavoro, il fordismo, il lavoro sessuale, la relazione tra lavoro femminile e religione, situandoli in specifici contesti socio-culturali e temporali.

3 La precarietà lavorativa femminile nell'Italia del Terzo Millennio (Raffaella Iorio) chiude temporalmente il dossier, mentre il rapporto tra genere e fordismo negli Stati Uniti degli anni Dieci/Venti (Bruno Walter Renato Toscano) ne costituisce il termine ad quem. Il dibattito sul lavoro sessuale (Arianna Pasqualini, Irene Vascelli) è analizzato nel lungo periodo, con un'attenzione al mutamento della figura della prostituta/sex worker, e attraverso una comparazione tra vari paesi europei (Germania, Svezia, Francia e, in misura più limitata, Italia). La relazione tra lavoro femminile, emancipazione e religione musulmana è invece indagata nel contesto medio-orientale e del nord Africa (Arianna Colella).

4 Nell'indagare temi, fenomeni, dibattiti così vari e contesti così lontani, i saggi hanno adottato una prospettiva comune, hanno riportato al centro il punto di vista femminile, come nel caso del dibattito sul lavoro sessuale o sulla precarietà, o i rapporti di genere, come nei contesti lavorativi culturalmente e socialmente mascolinizzati della fabbrica Ford o dell'area medio-orientale e maghrebina.

5 Valendosi dell'interpretazione gramsciana dei concetti di "fordismo" e "questione sessuale", Toscano ha condotto un'analisi originale sui rapporti di genere interno ed esterni alla fabbrica Ford, enucleando tre diverse figure di donne rimaste ai margini della storiografia su Ford e il Fordismo: l'operaia, la casalinga, consumatrice.

6 La centralità attribuita da Vascelli e Pasqualini al pensiero delle lavoratrici del sesso $\mathrm{e}$ alle elaborazioni dei movimenti femminili che a vario titolo e in varie epoche si sono occupati del lavoro sessuale appare utile a ripensare la stessa concettualizzazione di prostituzione, ancora dominante nella legislazione e nel dibattito pubblico.

7 Il ruolo del lavoro femminile per l'emancipazione delle donne musulmane in MedioOriente e Nord Africa è indagato in relazione allo status giuridico della donna non solo nel mercato del lavoro, ma anche nella famiglia. Mutamenti e persistenze costituiscono la cifra di una condizione, quella della lavoratrice musulmana, ancora poco conosciuta nel contesto italiano e che Colella contribuisce a disvelare.

8 La riflessione femminile sulla precarietà, al centro del saggio di Iorio, si coniuga con una disamina delle risposte inedite che proprio le donne hanno messo in campo negli anni della crisi globale. Il "ritorno a casa" e il "ritorno alla terra" sono proposte e 
interpretate non come occasioni mancate bensì come scelte alternative per una migliore conciliazione dei tempi di vita e lavoro.

\section{AUTORI}

\section{ELOISA BETTI}

Eloisa Betti si laurea (con lode) nel 2004 presso l'Università di Bologna in Storia Contemporanea, con una tesi in Storia del lavoro dal titolo Mutamenti nei rapporti di lavoro in Italia dalla crisi degli anni '70 alla flessibilità, alla quale viene attribuita la dignità di stampa. Nel 2011 consegue il dottorato di ricerca in Storia d'Europa presso la stessa università, discutendo la tesi Donne e precarietà del lavoro nell'industria Bolognese dagli anni Cinquanta alla crisi degli anni Settanta. Nel 2012 è risultata vincitrice di un assegno di ricerca dal titolo Donne, lavoro, diritti e politiche di genere a Bologna 1945-1992 (tutor prof.ssa Fiorenza Tarozzi), attualmente in corso di svolgimento. Ł̀ cultrice della materia presso il Dipartimento di Storia Culture Civiltà dell'Università di Bologna per i seguenti insegnamenti: Storia del lavoro (dal 2006); Storia delle donne e dell'identità di genere (dal 2014); Storia sociale (dal 2016) e presso la Scuola di Lingue e Letterature, Traduzione e Interpretazione dell'Università di Bologna per Storia contemporanea (dal 2014).

URL: < https://www.unibo.it/sitoweb/eloisa.betti2/cv >

\section{MARIA PIA CASALENA}

Maria Pia Casalena si è laureata in Storia contemporanea all'Università di Bologna, dove ha successivamente conseguito il dottorato di ricerca in Storia d'Europa (in cotutela con l'Université de Paris I-Panthéon Sorbonne). Borsista post-dottorato e assegnista di ricerca presso il Dipartimento di Discipline storiche dell'Università di Bologna, ha tenuto per un biennio l'insegnamento di Storia delle donne e di genere. Si occupa di storia sociale e culturale del secolo XIX, in particolare di storia del Risorgimento italiano e di storia delle donne. Ha pubblicato i seguenti volumi: Scritti storici di donne italiane. Bibliografia 1800-1945 (Firenze 2003); Per lo Stato per la Nazione. I congressi degli scienziati in Francia e in Italia (1830-1914) (Roma 2007); (con Francesca Sofia) Cher Sis. Scritture femminili nella corrispondenza di Sismondi (Firenze 2008); Biografie. La scrittura delle vite in Italia tra politica società e cultura (1796-1915) (Milano 2012); Le italiane e la storia: un percorso di genere nella cultura contemporanea (Milano 2016); Libertà progresso e decadenza. La storiografia di Sismondi (Bologna 2016); Eroi in bilico: il Risorgimento nei dizionari biografici del Novecento (Roma 2018).

URL: < https://www.unibo.it/sitoweb/mariapia.casalena/cv > 